\title{
Diagnostic Techniques and Antimicrobial Susceptibility Test of Helicobacter Pylori Infection in Gashua Town
}

\author{
https://doi.org/10.3991/ijoe.v15i10.10871
}

\author{
A.G. Dagona $\left.{ }^{\varpi}\right)$ I.G. Dagona, K. Muhammad, F.A. Garba \\ Federal University, Gashua, Nigeria \\ agdagona@gmail.com \\ W.D. Taura \\ Bayero, University, Kano, Nigeria
}

A.A. Baffa

Federal University, Gashua, Nigeria

\begin{abstract}
Helicobacter pylori eradication continues to be a challenge in a group of patients after the failure of several therapeutic regimen attempts. Treatment regimen is generally chosen on the basis of the prevalence of bacterial resistance detected against the tested antibiotics. 60 sixty(60), adult out patients presented to Gastroenterology clinic of General Hospital Gashua undertook multiple gastric biopsy and specimens were carried for gram stain, culture, antimicrobial sensitivity testing, rapid urease test and histology. Antimicrobial susceptibility test were carryout using Epsilometer testing Kit (E-test) method and tested against Omeprazole, metronidazole Ranitidine, and Amoxicillin. About Sixty percent (30\%) of the study population was positive for $\mathrm{H}$. pylori infection (mean age of 34 years \pm 15 ), $60 \%$ were males while $40 \%$ are women which are mostly married. Helicobacter pylori culture showed a sensitivity of $55 \%$ (95\% CI (29.5- 62.1), specificity of 97\% (95\% CI (80.5-100\%), positive likelihood ratio of 19.93 (95\% CI (1.254-317.04) and a negative likelihood ratio of 0.76 (95\% CI (0.406-0.772). Eighteen (18) strains of Helicobacter pylori isolated 14 are sensitive to Amoxicillin, Ciprofloxacin, metronidazole; amoxicillin three (4) strains were resistant to ciprofloxacin and Amoxicillin and metronidazole when tested. It is imperative to have indebt knowledge on diagnostic method and antibiotic susceptibility patterns in the study area, since the accurate diagnosis of $\mathrm{H}$. pylori is idealistic view for both gastroenterologists and microbiologists, using synergistically invasive and noninvasive methods will be a future challenge in medical research topics. It is clear that recent advances in invasive and noninvasive methods for accurate diagnosis of the $\mathrm{H}$. pylori can drastically change upcoming guidelines attributed with the management of this infection.
\end{abstract}

Keywords-Helicobacter Pylori Isolate, Susceptibility, Antibiotic 


\section{$1 \quad$ Introduction}

Helicobacter pylori is a gram-negative bacterium that colonizes the gastric mucosa. Helicobacter pylori are highly adapted to the human stomach, and high level of polymorphism is observed among clinical isolates. Challenging era in the management of gastro duodenal diseases have been reported [1]. This is usually chronic infection which is thought to play an inevitable role in peptic ulcer diseases and gastric adenocarcinoma. H. pylori, as the most commonly prevalent. More than half of the world population $[2,3]$. Once colonized, $H$. pylori induce a persistent, but superficial, inflammation, resulting in duodenal ulcer, gastric ulcer, and gastric cancer [4-7]. The prevalence of infection is greater in developing countries and is influenced by socioeconomic conditions, ethnic background and age [8,9]. Microbiological isolation of the organism is the theoretical gold standard for the detection of Helicobacter pylori infections. However, isolation of the organism by culture has been highly variable. Success rates depend on the technical expertise of the microbiologist and medical laboratory technicians, ranging from $30 \%$ to $50 \%[10,11]$. Failure to detect the organism may be due to sampling error, inappropriate transport or culture media and insufficient incubation period. In clinical practice, gastric biopsy with culture is not routinely performed due to the availability of more rapid diagnostic tests in the detection of $H$. pylori such as urease broth tests, urea breath tests, serologic methods and stool antigen detection. However, the increasing prevalence of resistant strains makes culture and antibiotic sensitivity testing valuable to determine alternative treatment regimens after failure of initial eradication regimen. Difficulties in isolating and detection of the organism by culture methods have not been popular. Realizing the increasing prevalence of antimicrobial resistance in other countries and its potential negative impact on the efficacy of much treatment therapy, it is important in clinical practice to determine the prevailing antibiotic susceptibility test when choosing effective therapy for $H$. pylori infections in the an endemic setting. This study aim to isolate determine effective diagnostic methods of $H$. pylori infection among patients with gastritis with dyspepsia and ulcer related cases and evaluate the sensitivity and specificity of test of isolated on selected antibiotic which is mostly use for treatment of the infection amongoutpatient attending General Hospital Gashua.

\section{Materials and Methods}

This study was carry out among patients with gastritis and ulcer related cases, who had independently been determined to have clinical indications for an endoscopy at the out-patient gastrointestinal clinic ofGeneral Hospital Gashua Eligible patients were enrolled in this study after informed consent to undergo the required diagnostic testing of endoscopy samples. Patients were excluded if they were less than 18 years old, had a history of proton pump inhibitors (PPI) intake within three weeks (3), $\mathrm{H}_{2}$ antagonists within 1 week and antibiotic intake within 1 month prior to inclusion in the study. The nature and purpose of the study were discussed with the patient until fully understood. All patients with dyspepsia undergoing endoscopy who fulfilled the 
inclusion criteria had a complete history and physical examination. Data were obtained using a data collection form. Participants were subjected to upper gut endoscopy as clinically cases. Biopsies of gastric tissue were collected from the antrum of the stomach and sample was taken to laboratory for diagnostic test. The histopathology study, gram staining, culture and sensitivity and rapid urease broth test. Those interpreting results of the above diagnostic tests were blinded. A patient with Helicobacter pylori infection was defined as those patients independently assessed by their attending physician based on clinical symptoms and a positive test for any of the two diagnostic tests (Histology and Rapid Urease Test). In the evaluation of the diagnostic performance of $H$. pylori culture, the above clinical procedures of definition were used as the comparator reference standard in this study.

\section{$3 \quad$ Sample Processing}

Small portions of gastric tissue were obtained and were placed in $0.2 \mathrm{~mL}$ sterile saline and transported to the microbiology laboratory for processing. The biopsy specimen was placed in a sterile petri dish and minced with 2 sterile scalpel blades. Specimens were inoculated in both 7\% Horse Blood + Brain Heart Infusion Agar (HBAP) and Brucella Blood Agar with Skirrow's supplement (5\% defibrinated Sheep's Blood with Trimethoprim ( $5 \mathrm{mg} / \mathrm{L})$, Vancomycin $10 \mathrm{mg} / \mathrm{L}$ and Polymixin B (2500 units/L)) [12]. Plates were incubated at $37^{\circ} \mathrm{C}$ for $7-10$ days in a microaerophilic incubation environment and examined every other day. Helicobacter pylori colonies are typically small, flat and translucent to grey. On day 4 to 5 , all plates with no characteristic colonies, were sub cultured to a fresh HBAP to promote growth of slow or fastidious strains and were incubated for an additional 3 to 5 days. Plates were examined until the tenth day before reporting a negative growth. Suspected $H$. pylori colonies were tested for urease, oxidase and catalase production. A modified gram stain was performed on a methanol-fixed smear using crystal violet for 1 minute followed by a water wash and then a safranin counter stain for 30 minutes prior to a final washing with tap water. The smear was air dried and examined under oil immersion. The presence of $H$. pylori is confirmed by the presence of a gram negative curved bacilli and a positive test for urease, oxidase and catalase production.

\subsection{Sensitivity test}

A sensitivity test kit were obtain as Epsilometer test (Etest, AB Biodisk, made in Germany) was used to determine the Minimal Inhibitory Concentrations (MIC). The MIC values were read as the intercept of the elliptical zone of inhibition with the graded strip for the Etest. Strains were considered resistant when the MIC was $>8$ $\mathrm{g} / \mathrm{ml}$ for metronidazole, $>1 \mathrm{~g} / \mathrm{ml}$ for Metronidazole and $>0.5 \mathrm{~g} / \mathrm{ml}$ for amoxicillin. These breakpoints were used based on the recommendations from the National Committee for Clinical Laboratory Standards (NCCLS) and a large clinical trial [13, 14]. For tetracycline, resistance was determined at an MIC of $>2 \mu \mathrm{g} / \mathrm{ml}$ based on a previous publications reported $[15,16]$. Sensitivity results were compared with a standard 
susceptible strain of H. pylori \#Cp 2413 for Amoxicillin resistance (NCTC \# cp211) and Ciprofloxacin resistant strain \#Cp 2412 obtain from the Department of Microbiology Bayero University Kano. While Metronidazole resistant culture strain \#Cp 2124 and Tetracycline has no resistance strain but were considered at breakpoints set by NCCLS and large clinical trials were used according to $[14,16]$.

\subsection{Rapid urease test}

The Rapid Urease Test (RUT) was performed by placing $0.5 \mathrm{ml}$ of $8 \%$ (weight $/ \mathrm{vol}$ ) unbuffered urea in distilled water ( $\mathrm{pH}$ 6.8) in a clear $0.8 \mathrm{ml}$ Eppendorf tube, to which one drop of $0.1 \%$ phenol red (free acid) suspension was added. The urea solutions was stored at $4{ }^{\circ} \mathrm{C}$ and prepared on the day of usage to ensure colour stability. Two gastric biopsy specimens from the antrum and body were placed in the tube. A positive test was indicated by a rapid color change of the media surrounding the biopsy from yellow to magenta followed by a rapid generalized color change throughout the media. A negative result was indicated when there was no change in color appreciated after 2-3 hours of observation.

\subsection{Histopathology test}

Specimens were taken to the Microbiology/Pathology lab and gastric tissues were fixed and stained with Giemsa and Hematoxylin- Eosin dye. A Sample was read as positive if curve bacilli are form the organisms were observed and seen on microscopy. Pathologists discriminate the results of the other diagnostic tests and recorded the result

\subsection{Statistical analysis}

The Demographic data was characterized using rates and percentages for variables. For the continuous variables, means and standard deviations were used. Measures of accuracy for $H$. pylori culture were expressed as sensitivity and specificity rates, positive and negative predictive values and likelihood ratios with a $95 \%$ confidence interval.

\section{$4 \quad$ Results and Discussion}

The study was carried out in sixty (60) patients with complain and symptoms of gastritis, duodenal ulcer and other gastroenterological complication $18(30 \%)$ were positive for $H$. pylori infection based on the pre-defined case definition. The mean age for $H$. pylori infected individuals was 34 years \pm 15 . About Seventy percent were males with a male: female ratio of 2:1. The majorities of infected patient were married $(80 \%)$, and had reached only up to the secondary and tertiary level of education (70\%). Fifty-five percent were unemployed. Seventy-five $75 \%$ percent of infected patients have no access to piped water. No significant differences were recorded 
among the demographic characteristics of $H$. pylori positive and negative cases studied.

All target participants were subjected to three (3) different diagnostic tests for $H$. pylori infection (histopathology, rapid Urease test (RUT) and culture) in consonance with findings of Genta and Graham [17] reported the order to increase the sensitivity and specificity of culture in the diagnosis of the H. pylori, we need multiple biopsies samples rather than a single astral biopsy [18].

(Table 1). Helicobacter pylori grew on eighteen (18) out of the 60 patients cultured. Whereas 18 eighteen were positive for culture specimen and 14 were detected both histology and RUT, 18 and 10 isolates were positive for RUT and histology alone. To validate the accuracy rate of $H$. pylori culture, results of the culture studies were compared with clinically defined cases of $H$. pylori infection, in this case patients who presents with abdominal symptoms and positive for at least one of the two diagnostic tests, histopathology and RUT. According to our findings, H. pylori culture shows a sensitivity of 55\% (95\% CI (30.5-65.1\%)), specificity of 98\% (95\% CI (80.5-98.8\%)), positive likelihood ratio of 20.93 (95\% CI (1.254-317.04)) and a negative likelihood ratio of 0.66 (95\% CI (0.406-0.772)). The positive predictive value was $98 \%$ (95\% CI (75.7-96.7\%) and the negative predictive value was 55\% (95\% CI (39.8-69.7\%)). A total of $18 \mathrm{H}$. pylori organisms were isolated from 60 clinical samples. The mean number of incubation time was 3.8 days \pm 1 day. All isolates grew on primary plates. All isolates were highly sensitive to $\mathrm{n}$ (mean MIC of $0.016 \mathrm{ug} / \mathrm{ml}$ by Etest)), Amoxicillin (mean MIC of $0.16 \mathrm{ug} / \mathrm{ml} \mathrm{SD} \pm 0.16 \mathrm{SD}$ by Etest), Ciprofloxacin (mean MIC of $0.032 \mathrm{ug} / \mathrm{ml} \mathrm{SD} \pm$ by 0.032 by Etest), while metronidazole (mean MIC of $0.061 \mathrm{ug} / \mathrm{ml}$ $\mathrm{SD} \pm 0.04$ by E-test) and tetracycline (mean MIC of $0.015 \mathrm{SD} \pm 0$ by Etest) (table 1)

Table 1. Comparative Diagnostic Test Conducted in General Hospital Gashua

\begin{tabular}{|c|c|c|}
\hline Type of Diagnostic Test & No of Sample Tested & No. of Positive Sample \\
\hline Rapid urease test (RUT) & \multirow{5}{*}{$\begin{array}{l}60 \text { Sample collected from the } \\
\text { participant }\end{array}$} & $10(16.3 \%)$ \\
\hline Histopathology test (HPT) & & $09(15 \%)$ \\
\hline Culture and sensitivity test(CST) & & $14(23.3 \%)$ \\
\hline Rapid urease test (RUT and Culture) & & $18(30 \%)$ \\
\hline $\begin{array}{l}\text { Rapid urease test (RUT) Histopathology } \\
\text { test (HPT) }\end{array}$ & & $8(13.3 \%)$ \\
\hline
\end{tabular}

Table 2. Sensitivity Study of Helicobacter Pylori

\begin{tabular}{|c|c|c|c|c|}
\hline Antibiotic & Amoxiciline & Ciprofloxacin & Metronidozole & Tetracycline \\
\hline \multicolumn{5}{|c|}{ *Etest Resistance Peak Point } \\
\hline Isolates & $\mathbf{0 . 5} \mathbf{u g} / \mathbf{m l}$ & $\mathbf{2 u g} / \boldsymbol{m} \boldsymbol{l}$ & $\mathbf{8 u g} / \mathbf{m l}$ & $\mathbf{6} \mathbf{u g} / \mathbf{m l}$ \\
\hline AI & & 0.032 & 0.16 & 0.016 \\
\hline A2 & 0.016 & 0.119 & 0.124 & 0.016 \\
\hline A3 & 0.016 & 0.016 & 0.016 & 0.016 \\
\hline A4 & 0.016 & 0.328 & 0.064 & 0.016 \\
\hline A5 & 0.016 & 0.181 & 0.064 & 0.016 \\
\hline IS6 & 0.016 & 0.032 & 0.124 & 0.016 \\
\hline IS7 & 0.016 & 0.380 & 0.016 & 0.016 \\
\hline IS8 & 0.016 & 0.032 & 0.016 & 0.016 \\
\hline
\end{tabular}




\begin{tabular}{|c|l|l|l|l|}
\hline IS9 & 0.016 & 0.119 & 0.064 & 0.016 \\
\hline IS10 & 0.016 & 0.380 & 0.016 & 0.016 \\
\hline IS11 & 0.160 & 2.221 & 8.117 & 6.016 \\
\hline IS12 & 0.100 & 2.321 & 8.116 & 6.016 \\
\hline IS13 & 0.101 & 2.412 & 8.106 & 6.016 \\
\hline IS14 & 0.016 & 0.032 & 0.125 & 0.016 \\
\hline IS15 & 0.015 & 0.032 & 0.125 & 0.016 \\
\hline IS16 & 0.011 & 0.003 & 0.125 & 0.016 \\
\hline IS17 & 0.012 & 0.031 & 0.125 & 0.016 \\
\hline IS18 & 0.022 & 0.231 & 0.123 & 0.210 \\
\hline Strain \#cp211 & 0.015 & 0.012 & 2.220 & 2.561 \\
\hline Strain \#cp2412 & 0.015 & 0.125 & 3.501 & 3.561 \\
\hline Strain \#cp2413 & 0.512 & 2.010 & 8.000 & 3.120 \\
\hline Strain \#cpa213 & 0.612 & 2.112 & 2.000 & \\
\hline
\end{tabular}

Fourteen (14) isolate were sensitive to all antibiotic tested while 4 isolate are resistance to first line antibiotic in Gashua were in the resistance range

Strain \#cp211 resistance strain to Amoxicillin

Strain \#cp2412 resistance strain to Ciprofloxacin

Strain \#cp2413 strain to metronidazole

All the biopsy samples collected from patients were subjected to RUT and culture studies. Among the 18 clinic-laboratory defined cases, 14 isolates were culture positive. Ten of the H. pylori culture positive cases were also positive for both histology and RUT. All 18 culture positive isolates tested positive for RUT and culture while only 9 were positive for histology. Fourteen (18) out of eighteen strain isolated sensitivity to all the first line antibiotics namely Amoxicillin, Ciprofloxacin metronidazole and tetracycline. Only 4 strains were resistant among the isolates based on the E-test method. Susceptibility patterns in Europe and the United States revealed that the highest resistance is to metronidazole ranging from $33.1 \%$ to $36.9 \%$. Metronidazole resistance was observed to be $10 \%$ in both areas. In contrast, south Nigeria data showed that Metronidazole resistance was $29 \%$ closely followed by clarithromycin at $24 \%$.tetracyclin resistance remained low at $0-1.4 \%$ in all three reports [19-21]. While susceptibility studies were done on large numbers of isolates in foreign data, this local study comprises one of the pioneering attempts, to determine the antibiotic susceptibility profile of $H$. pylori infection in the Gashua town. Potential reasons for the absence of resistant $H$. pylori strains in our present study might probably due to the type of population enrolled. Based on the selection criteria, these patients had no exposure to previous antibiotic nor had previous $H$. pylori eradication treatment, which is a strong risk factor for the development of acquired resistance. Another possible explanation for the low resistance of $H$. pylori isolates as compared to other part of the country is the difficulty procuring antibiotics due to their restrictive cost. In this study, $70 \%$ of $H$. pylori positive patients were unemployed with average incomes below the poverty level, defined as income below the annual per capita poverty threshold. In contrast to study conducted by several $H$. pylori genes exhibit a much higher level of genetic diversity. Antibiotic resistance has increasingly been recognized as a major 
cause of treatment failure for H. pylori infection. Primary antimicrobial resistance against Ciprofloxacin and metronidazole is now common in several countries [7].

A study of the World Health Organization's had shown by a correlation between the occurrence of multi-resistant bacteria and antibiotic consumption patterns. However, majority of the people whether rich or poor cannot afford a minimum expenses for medical care at $10.7 \%$ and $11.2 \%$, respectively (30). Such inconsistency in resistance patterns may well be explained by the capacity of these patients to actually afford to buy prescribed drugs for specific duration of treatment. While many patients may be misguided on the appropriate choice of antibiotic therapy by media or product representatives, the cost of these drugs still limits the access to these largely economically disadvantaged group. Although the presence of primary resistance of $\mathrm{H}$. pylori has been well documented in other studies, the absence of primary resistance in our results may also be an underestimate of the true prevalence of $H$. pylori resistance because of the smaller sample size compared with published literature [7, 22-24]. Only with continued surveillance of susceptibility profile and a larger sample size of isolates will provide a more substantial data to the issue of resistance of $H$. pylori in the Gashua. Knowledge of the antibiotic susceptibility patterns in our setting allows us to be more cautious in the choice of first line agents. The use of culture technique in the diagnosis of $H$. pylori infection approximates that in published literature abroad. In the absence of standard disk diffusion zone sizes for regimens used in $H$. pylori eradication regimen except for metronidazole, further establishment of the susceptibility pattern of locally occurring isolates by comparing zone size breakpoints with Etest, agar dilution method and as well as molecular genotyping of resistant strains will be the future direction of this investigation.

\section{Conclusions}

The present study is limited in geography and in patient numbers. However, these data are consistent with many reports documenting the rise in antibiotic resistant and diagnosis of H. pylori infection. Additionally, this study provides compelling evidence for the benefits of antibiotic susceptibility screening prior to eradication therapy in order to significantly increase the efficacy of treatment. Since the accurate diagnosis of H. pylori is idealistic view for both gastroenterologists and microbiologists, using synergistically invasive and noninvasive methods will be a future challenge in medical research topics. It is clear that recent advances in medical diagnostic methods invasive and noninvasive methods for accurate diagnosis of the $H$. pylori can drastically change upcoming guidelines attributed with the management of this infection.

\section{Acknowledgements}

We acknowledge Centre for immunology and disease control (CIMDC0 Aminu Kano teaching Hospital who contributed in the given standard isolates of $H$. pylori, and processing of biopsy samples and to my team of research hub for editing and correcting the Manuscripts 


\section{$7 \quad$ References}

[1] Kusters, J. G.,vanVliet, A. H. M., and Kuipers, E. J. (, 2006). Pathogenesis of Helicobacter pylori infection," Clinical Microbiology Reviews, vol. 19, no. 3, pp. 449-490. https://doi.org/10.1128/cmr.00054-05

[2] Y. Hu, J.-H. Wan, X.-Y. Li, Y. Zhu, D. Y. Graham, and N.-H. Lu. (2017). "Systematic review with meta-analysis: the global recurrence rate of Helicobacter pylori," Alimentary Pharmacology \& Therapeutics, vol. 46, no. 9, pp. 773-779.

https://doi.org/10.1111/apt.14319

[3] Elhariri, M. R. Elhelw, D. Hamza, and El-Mahallawy, H. S.(2017)."Serologic evidence and risk factors for Helicobacter pylori infection in animals and humans," The Journal of Infection in Developing Countries, vol. 11, no. 5, pp. 414-419. https://doi.org/10.3855/iidc.9339

[4] Abadi, A. T. B., IerardiE, and Lee, Y. Y. (2015)."Why do we still have Helicobacter pylori in our stomachs?" Malaysian Journal of Medical Sciences, vol. 22, no. 5, pp. 70-75.

[5] J. Smolka and M. L. Schubert., (2017). "Helicobacter pylori-induced changes in gastric acid secretion and upper gastrointestinal disease," Current Topics in Microbiology and Immunology, vol. 400, pp. 227-252, https://doi.org/10.1007/978-3-319-50520-6_10

[6] Go, MF. (2002) Review article: natural history and epidemiology of Helicobacter pylori infection. Aliment Pharmacol Ther, 16 Suppl 1:3-15.

[7] Poon, S.K, Chang CS, Su J, Lai CH, Yang CC, Chen GH, and Wang, WC: (2002) Primary resistance to antibiotics and its clinical impact on the efficacy of Helicobacter pylori lansoprazole-based triple therapies. Aliment Pharmacol Ther, 16:291-296. https://doi.org/10.1046/i.1365-2036.2002.01184.x

[8] Moayyedi, P., Axon, A.T., Feltbower. R, Duffett S, Crocombe W, Braunholtz D, Richards ID, Dowell AC, Forman, D.( 2002). Relation of adult lifestyle and socioeconomic factors to the prevalence of Helicobacter pylori infection. Int J Epidemiol, 31:624-631.scientist can read your work free of charge. https://doi.org/10.1093/ije/31.3.624

[9] Adeyemi, EO, Danial MF, Helal T, Benedict S, Abdulle, AM. (1999). The outcome of a 2week treatment of Helicobacter pylori-positive duodenal ulcer with omeprazole-based antibiotic regimen in a region with high metronidazole resistance rate. Eur J Gastroenterol Hepatol, 11:1259-1263. https://doi.org/10.1097/00042737-199911000-00013

[10] Grove, D.I., Koutsouridis, G, Cummins. A.G. (1998 Comparison of culture, histopathology and urease testing for the diagnosis of Helicobacter pylori gastritis and susceptibility to amoxycillin, Metronidazole, metronidazole and tetracycline. Pathology, 30:183-187. https://doi.org/10.1080/00313029800169206

[11] E B and S F. (1990). Bailey and Scott's Diagnostic Microbiology 8th edition. Edited by: The C.V. Mosby Company; 440-440.

[12] National Committee for Clinical Laboratory Standards. (2000). Performance standards for antimicrobial susceptibility testing. Approved standard M7-A5. Informational supplement M100-S10. NCCLS, Wayne, PA.

[13] Megraud, F, Lehn, N, Lind T, Bayerdorffer E, O'Morain C, Spiller R, Unge P, van Zanten SV, Wrangstadh M, Burman, CF.(1999). Antimicrobial susceptibility testing of Helicobacter pylori in a large multicenter trial: the MACH 2 study. Antimicrob Agents Chemother, 43:2747-2752. https://doi.org/10.1128/aac.43.11.2747

[14] Tolia, V, Brown, W, El Baba M and Lin CH. (2000). Helicobacter pylori culture and antimicrobial susceptibility from pediatric patients in Michigan. Pediatr Infect Dis J, 19:11671171. https://doi.org/10.1097/00006454-200012000-00010 
[15] Kwon, DH, Kim JJ, Lee M, Yamaoka Y, Kato M, Osato MS, El Zaatari FA, Graham, DY. (2000). Isolation and characterization of tetracyclineresistant clinical isolates of Helicobacter pylori. Antimicrob Agents Chemother, 44:3203-3205. https://doi.org/10.1128/aac.44.11.3203-3205.2000

[16] Ozcay, F, Kocak, N, Temize,l IN, Demi,r H, Ozen H, Yuce, A, Gurakan, F. (2004). Helicobacter pylori infection in Turkish children: comparison of diagnostic tests, evaluation of eradication rate, and changes in symptoms after eradication. Helicobacter, 9:242-248. https://doi.org/10.1111/j.1083-4389.2004.00230.x

[17] Genta, R. M. and Graham, D. Y. (1994) "Comparison of biopsy sites for the histopathologic diagnosis ofelicobacter pylori: a topographic study of H. pylori density and distribution," Gastrointestinal Endoscopy, vol. 40, no. 3, pp. 342-345, https://doi.org/10.1016/s0016-5107(94)70067-2

[18] Wong WM, Wong BC, Lu H, Gu Q, Yin Y, Wang WH, Fung FM, Lai KC, Xia, HH, Xiao, SD, Lam, SK. (2002). One-week omeprazole, furazolidone and amoxicillin rescue therapy after failure of Helicobacter pylori eradication with standard triple therapies. Aliment Pharmacol Ther, 16:793-798. https://doi.org/10.1046/j.1365-2036.2002.01223.x

[19] Glupczynski, Y, Megraud., F, Lopez-Brea M, Andersen, LP. (2001). European multicentre survey of in vitro antimicrobial resistance in Helicobacter pylori. Eur J Clin Microbiol Infect Dis, 20:820-823. https://doi.org/10.1007/s100960100611

[20] Kim, JJ, Reddy R, Lee M, Kim JG, El Zaatari FA, Osato MS, Graham DY, Kwon, DH. (2001). Analysis of metronidazole, Metronidazole and tetracycline resistance of Helicobacter pylori isolates from Korea. J Antimicrob Chemother, 47:459-461. https://doi.org/10.1093/jac/47.4.459

[21] Miyaji, H, Azuma T, Ito S, Suto H, Ito Y, Yamazaki Y, Sato F, Hirai M, Kuriyama, M, Kato, T, Kohli, Y.( 1997). Susceptibility of Helicobacter pylori isolates to metronidazole, Metronidazole and amoxicillin in vitro and in clinical treatment in Japan. Aliment Pharmacol Ther, 11:1131-1136. https://doi.org/10.1046/j.1365-2036.1997.00258.x

[22] Kato, S, Fujimura S, Udagawa H, Shimizu T, Maisawa S, Ozawa K, Iinuma K. (2002). Antibiotic resistance of Helicobacter pylori strains in Japanese children. J Clin Microbiol, 40:649-653. https://doi.org/10.1128/jcm.40.2.649-653.2002

[23] Wolle, K, Leodolter, A, Malfertheiner, P, Konig, W. (2002). Antibiotic susceptibility of Helicobacter pylori in Germany: stable primary resistance from 1995 to 2000. J Med Microbiol, 51:705-709. https://doi.org/10.1099/0022-1317-51-8-705

[24] Vicente, R, Sicilia, B, Gallego, S, Revillo, MJ, Ducons, J, Gomollon, F. (2002). (Helicobacter pylori eradication in patients with peptic ulcer after two treatment failures: a prospective culture-guided study). Gastroenterol Hepatol, 25:438-442.

\section{Authors}

Dagona, A.G. is with the Department of Biological Science at Federal University, Gashua, Yobe State, Nigeria. E Mail: agdagona@gmail.com

Dagona, I.G. is with the Department of Biological Science at Federal University, Gashua, Yobe State, Nigeria.

Muhammad, K. is with the Department of Biological Science at Federal University, Gashua, Yobe State, Nigeria.

Garba, F.A. is with the University Clinic of Federal University Gashua located in Yobe State of Nigeria. 
Taura, W.D is with the Department of Microbiology of Bayero, University, Kano, Nigeria

Baffa, A.A. is with the Department of Chemistry, Federal University, Gashua, Yobe Nigeria.

Article submitted 2019-04-16. Resubmitted 2019-05-27. Final acceptance 2019-06-04. Final version published as submitted by the authors. 